\title{
Foot function and strength of patients with diabetes grouped by ulcer risk classification (IWGDF)
}

\author{
Jane S. S. P. Ferreira' ${ }^{1}$, João P. Panighel ${ }^{1}$, Érica Q. Silva ${ }^{1}$, Renan L. Monteiro ${ }^{1,2}$, Ronaldo H. Cruvinel Júnior ${ }^{1}$ \\ and Isabel C. N. Sacco ${ }^{1 *}$ (B)
}

\begin{abstract}
Background: The stratification system from the International Working Group on the Diabetic Foot (IWGDF) was used to classify the participants as to the ulcer risk. However, it is not yet known what the classification groups' individual deficits are regarding sensitivity, function, and musculoskeletal properties and mechanics. This makes it difficult to design proper ulcer prevention strategies for patients. Thus, this study aimed to investigate the foot function, foot strength and health of people with diabetes mellitus (DM) — with or without DPN—while considering the different ulcer risk classifications determined by the IWGDF.
\end{abstract}

Methods: The subject pool comprised 72 people with DM, with and without DPN. The patients were divided into three groups: Group 0 (G0), which comprised diabetic patients without DPN; Group 1 (G1), which comprised patients with DPN; and Group 2 (G2), which comprised patients with DPN who had foot deformities. The health and foot function of the subjects' feet were assessed using a foot health status questionnaire (FHSQ-BR) that investigated four domains: foot pain, foot function, footwear, and general foot health. The patients' foot strength was evaluated using the maximum force under each subject's hallux and toes on a pressure platform (emed q-100, Novel, Munich, Germany).

Results: Moderate differences were found between G0 and G1 and G2 for the foot pain, foot function, general foot health, and footwear. There was also a small but significant difference between G0 and G2 in regards to hallux strength.

Conclusion: Foot health, foot function and strength levels of people with DM and DPN classified by the ulcer risk are different and this must be taken into account when evaluating and developing treatment strategies for these patients.

Keywords: Muscle weakness, Diabetic neuropathies, Diabetic foot, Ulcer

\section{Background}

Plantar ulcers present major challenges for people with diabetes mellitus (DM), with a prevalence ranging from $0.65 \%$ in individuals under 44 years old to $1.3 \%$ in individuals aged 75 years or older [1]; moreover, the ulcers

\footnotetext{
*Correspondence: icnsacco@usp.br

${ }^{1}$ Department of Physical Therapy, Speech, and Occupational Therapy, School of Medicine, University of São Paulo, Rua Cipotânea, 51-Cidade Universitária, São Paulo 05360-160, Brazil

Full list of author information is available at the end of the article
}

have higher incidences in developing countries [2] and a $41.5 \%$ chance of reocurrence [3].

Amongst the most common chronic complications of diabetes are neuropathies, affecting up to $50 \%$ of DM patients [4]. The diabetic polyneuropathy (DPN) is characterized by loss of protective sensation, which in turn contributes to the appearance of plantar ulcers [4]. According to IWGDF, the loss of protective sensation can be assessed with one of the following techniques: pressure perception using a Semmes-Weinstein $10 \mathrm{~g}$ 
monofilament or vibration perception using a $128 \mathrm{~Hz}$ tuning fork. When a monofilament or a tuning fork are not available, it is recommended to test the tactile sensation by lightly touching the tips of the toes with the tip of the index finger for 1-2 s [5]. The IWGDF Risk Stratification System classifies people with diabetes in four groups according to the ulcer risk. Group 0, which comprised patients without DPN; Group 1, which comprised patients with DPN; Group 2, which comprised patients with DPN and with foot deformities and/or vascular disease; and Group 3, which comprised patients with DPN and history of a foot ulcer or a lower-extremity amputation (minor or major) or end-stage renal disease [5].

Sensitivity changes are due to DPN [6-10], which begins with impairments to peripheral nerves and progresses to impairments to motor and autonomic nerves. DPN results in progressive vibratory, thermal, tactile, and proprioceptive sensitivity deficits [10]. In addition to affecting the integrity of neural structures, DPN also affects musculoskeletal structures, such as lower limb joints, the calcaneal tendon, and intrinsic foot muscles [11-17], resulting in the decreased strength of the musculature of the foot/ankle complex and affecting both extensor and flexor muscles [18].

In addition to plantar ulcers and DPN, type $2 \mathrm{DM}$ is associated with changes in joint collagen [19] that compromise patients' physical functions related to daily living activities [20]. These functional deficits are due to aging joint collagen; decreased proteoglycans; and altered collagen types in ligaments, cartilage, and synovial fluids, which increase the rigidity of joint structures [21]. These changes result from the non-enzymatic glycation of collagen [22], which results in the chronic accumulation of advanced glycation products $[23,24]$. The increased stiffness of the skeletal muscle lead to an increased injury risk [25], decreased range of motion and impaired function [26, 27]. Such morphological alterations, found in patients with DM and DPN, lead to foot deformities that cause structural and functional changes to the foot region [28]. These changes are responsible for increased pressure in certain plantar areas, a factor directly related to the formation of plantar ulcers $[3,7,9,11,29,30]$.

Within this context, some researchers have listed the prevention of ulcers as a priority in terms of the study of the diabetic foot $[31,32]$. To optimize research into care strategies for those affected, the IWGDF established ulcer risk classification [5]. The classification consists of a stratification of people with DM into different categories of risk in terms of developing ulcers; a patient's risk is determined through a clinical evaluation, the presence or absence of foot deformities, and the patient's history of foot ulcers [5]. However, it should be noted that studies related to the prevention of plantar ulcers in people with DPN are ongoing and have had fewer positive findings [31] than studies related to the treatment of plantar ulcers.

When treating and preventing complications in DM patients with DPN, it is crucial to consider the strength levels of the patients' lower limbs, which are their most affected body areas [33]. Reductions in lower limb strength can lead to negative consequences, such as reduced mobility and function, which affect walking, running, stair climbing, and other daily living activities [33]. Patients can lose the ability to perform physical exercises important for metabolic control, physical capacity maintenance, and quality of life [34]. Furthermore, the reduction of the foot-ankle strength are related to fall incidences in people with DM [35]. Thus, determining effective prevention strategies for such patients involves the characterization of the population in question in regards to deficits in sensitivity, musculoskeletal properties and mechanics; and function, the objective of this study was to investigate the foot function, strength, and health of people with DM, with or without DPN, based on the different ulcer risk classifications of the IWGDF.

\section{Methods}

This cross-sectional study was conducted in the National Association of Assistance to Diabetes from May 2016 to December 2016 using a random series of recruitment and evaluations performed within this period. It was approved by an ethics committee (Comitê de Ética em Pesquisa da Faculdade de Medicina da Universidade de São Paulo, protocol 075/16). In sum, 240 people who were diagnosed with either type 1 or $2 \mathrm{DM}$, with or without DPN, were invited to participate. From the 240 subjects, 168 did not consent or did not meet the inclusion criteria, which were: participants aged between 40 and 75 years old, diagnosed with type 1 or type $2 \mathrm{DM}$ and absence of active plantar ulcers, major vascular complications and severe nephropathy. The age range specified as an inclusion criteria was established considering that (1) our sample consisted of type 2 DM patients, (2) whose diagnosis occurred around 40-45 years old and (3) DPN is a chronic complication that starts approximately 10 to 15 years after the first diagnosis of DM [36]. In addition, the maximum age of 75 years was established because significant changes in muscle strength and tropism are present in older people and could have biased our results.

Patients were excluded if they had other neurological impairments, such as dementia; if they had other orthopedic impairments; if they were unable to give consistent information; and if they had active plantar ulcers, major vascular complications, severe retinopathy, or severe nephropathy. Although the IWGDF classifies DM patients into four categories [5], we have not included 
patients from Group 3, which comprised patients with DPN and history of foot ulcer or lower-extremity amputation (minor or major) or end-stage renal disease was not included in the study, first because of the nature of the recruitment setting that is a preventive care center and severe patients are not the target, second because if any minor or major amputation was present, it would refrain them to accomplish the strength assessment.

The final subject pool comprised 72 subjects, who were divided into three groups based on the ulcer risk classifications of the IWGDF [5] (Fig. 1): Group 0, which comprised patients without DPN; Group 1, which comprised patients with DPN; and Group 2, which comprised patients with DPN and with foot deformities and/or vascular disease.

To include and classify the subjects, DPN, vascular disease, and foot deformities were assessed. The subjects were screened for DPN using the fuzzy score software (http://www.usp.br/labimph/fuzzy/ingles/index.php). Fuzzy scoring is an rule-based expert system that supports the classification of DPN into different levels, based on severity [12,37]. The system has an adequate accuracy with an Receiver Operating Characteristic ROC curve area $=0.91$ ) for expert classifications [12]. The model combines the results of tactile sensitivity evaluations (conducted using a $10 \mathrm{~g}$ monofilament); the results of vibratory sensitivity evaluations (conducted using a tuning fork); and symptoms of DPN. Numerical values ranging from zero to 10 are produced that can be sorted into different classes. For this study, values above 2.5 were considered; such values indicated that DPN was present.

In addition, peripheral arterial disease was determined and classified using an ankle-brachial index; values of less than 0.5 indicated the presence of severe vascular disease; values ranging from 0.5 to 0.9 indicated the presence of vascular disease; and values ranging from 0.9 to 1.2 were considered normal [38]. Finally, the presence of deformities was determined through visual inspections conducted by trained physiotherapists. The inspections determined the presence of claw toes, hammer toes, flat foot, cavus foot, callosities, and/or hallux valgus. The presence of only one deformity was necessary for the classification of deformed feet. The subjects' demographic, anthropometric, and clinical characteristics, and the subjects' ulcer risk classifications, are shown in Table 1.

\section{Outcomes and assessments}

An initial assessment was conducted to determine whether the subjects met the inclusion criteria. Then, a second assessment was conducted using the fuzzy score software to evaluate the presence and classify the severity of DPN in patients. The presence of neuropathy was classified by symptoms using the Michigan Neuropathy Screening instrument. The greater the score, the greater the symptoms amount and thus the DPN severity (Table 1). The assessment protocol also comprised two subsequent evaluations: the foot health status assessment based on the Foot Health Status Questionnarie (FHSQBR) [39], which was completed in a quiet room by all participants, and measurements of the subjects' hallux and toe strengths, conducted using the emed q-100 pressure platform (Novel, Munich, Germany) (Fig. 1).

\section{Foot strength assessments}

The subjects' isometric foot strength was measured using the emed pressure platform; this method is a reliable way to assess foot muscle strength with Intraclass Correlation Coefficient between assessment sessions (ICC) greater than 0.92 [40]. Each subject stood and pushed down on the pressure platform three times, as hard as possible, with his or her hallux and toes from one side randomly chosen before his/her first trial. The mean value of all trials from one foot both was used for statistical purposes. The maximum force under the patient's hallux and toes, normalized by patient's bodyweight, were the outcomes of this measurement.

\section{Foot health and function assessments}

This study used a Brazilian Portuguese version of an (FHSQ-BR) that was translated and validated [39]. Section I evaluated foot health using four domains: foot pain, foot function, footwear, and general foot health. Section II referred to general health, and Section III collected general demographic data. Only scores from Section I were used because Section II examined general health. Each domain in Section I could result in a score ranging from zero to 100 points, where 100 was the best condition and zero was the worst condition. The scores were calculated using version 1.03 of FHSQ software (Care Quest, Queensland, Australia).

\section{Statistical analyses}

A sample-size calculation was performed using version 3.1 of the software G*Power [41]. For this calculation, it was assumed: a type I error $(\alpha)$ of $5 \%$, a power of $85 \%$, a large effect size with an $f$ of $0.40,3$ groups, and a statistical design of one-way ANOVA. A number of subjects (72) were reached. For an inferential statistical analysis, continuous data were presented as means and standard deviations, categorical data are presented in percentage, comparisons among groups (G0, G1, and G2) for the continuous data were made using one-way ANOVAs for each variable analyzed, and Chi-square tests were used for other categorical data. These analyses were made 


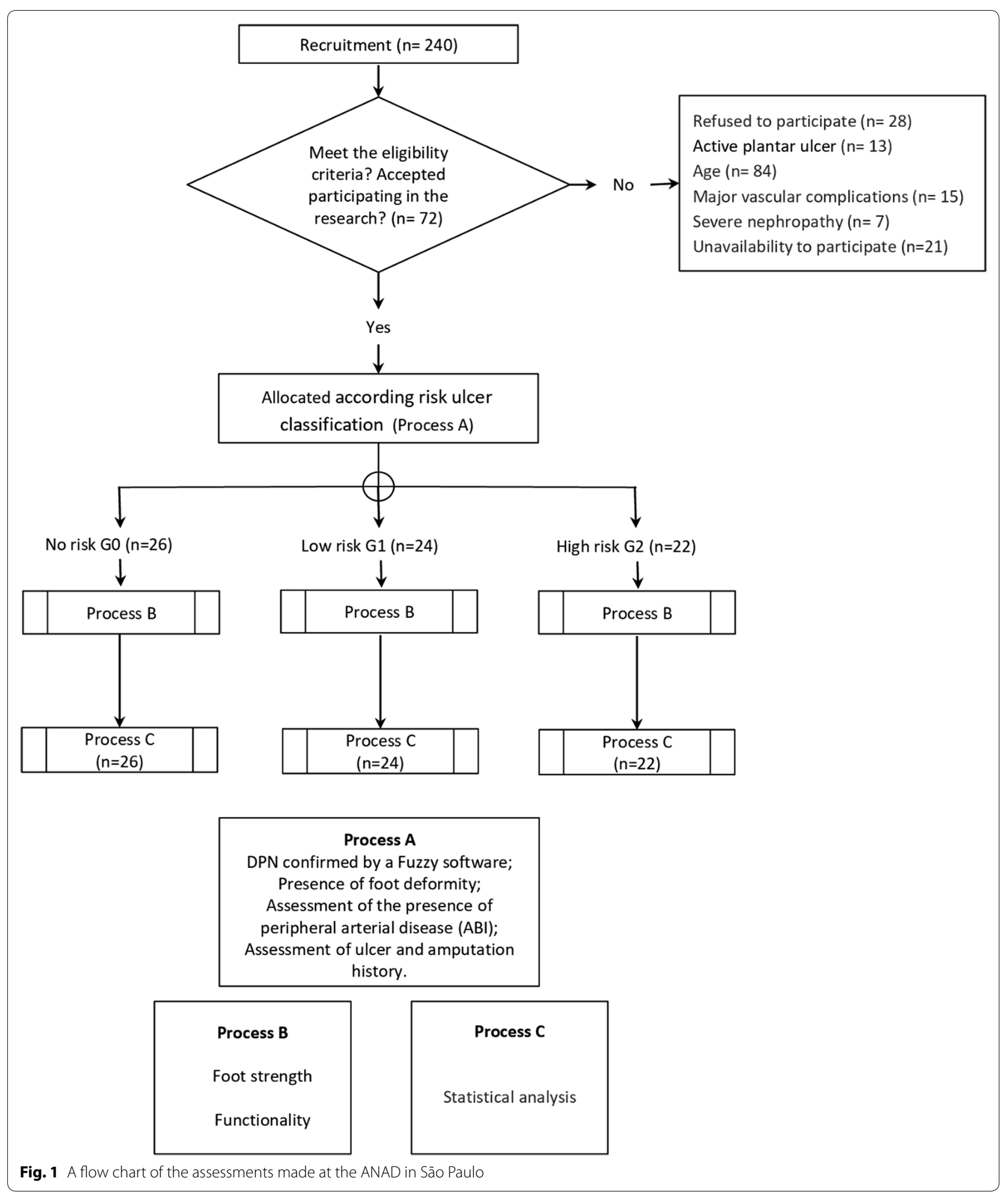

using version 12 of the software Statistica. Significant differences were considered with an $\alpha$ of $5 \%$.

\section{Results}

Regarding foot health status and foot function, there were moderate differences between the groups, especially 
Table 1 Mean and standard deviation and p-values of demographics, anthropometrics and clinical characteristics of the studied groups

\begin{tabular}{|c|c|c|c|c|}
\hline & $G 0(n=26)$ & $\mathrm{G1}(\mathrm{n}=24)$ & $G 2(n=23)$ & $\mathrm{p}$ \\
\hline Age (years) & $65.0 \pm 13.9$ & $63.0 \pm 16.1$ & $60.5 \pm 11.6$ & $0.522^{1}$ \\
\hline Males (\%) & 61.5 & 55.0 & 35.7 & - \\
\hline Body mass (kg) & $73.0 \pm 15.6$ & $72.0 \pm 11.9$ & $67.5 \pm 15.1$ & $0.872^{1}$ \\
\hline Height $(\mathrm{cm})$ & $162.0 \pm 11.4$ & $164.0 \pm 5.8$ & $158.0 \pm 10.8$ & $0.883^{1}$ \\
\hline Body mass index $\left(\mathrm{kg} / \mathrm{m}^{2}\right)$ & $32.5 \pm 4.9$ & $25.5 \pm 3.6$ & $27.9 \pm 3.7$ & ${ }^{*} 0.001^{1}$ \\
\hline Diabetes diagnosis (years) & $11.2 \pm 7.7$ & $15.9 \pm 11.8$ & $11.8 \pm 9.1$ & $0.234^{1}$ \\
\hline Presence of DPN (\%) & 0 & 100 & 100 & \\
\hline Tactile sensitivity loss [10 g monofilaments] (\%) & 0 & 100 & 100 & \\
\hline \multicolumn{5}{|l|}{ Vibratory sensitivity } \\
\hline Absent (\%) & 0 & 20.7 & 41.4 & $0.073^{2}$ \\
\hline Reduced (\%) & 0 & 79.3 & 58.6 & $0.759^{2}$ \\
\hline Presence of peripheral vascular disease (\%) & 0 & 0 & 8.7 & \\
\hline \multicolumn{5}{|l|}{ Presence of foot deformities (\%) } \\
\hline Claw toes & 0 & 0 & 52.0 & \\
\hline Hammer toes & 0 & 0 & 13.0 & \\
\hline Flat foot & 0 & 0 & 13.0 & \\
\hline Hallux valgus & 0 & 0 & 22.0 & \\
\hline
\end{tabular}

*Statistically significant difference

${ }^{1} \mathrm{p}$ values for the ANOVA tests

${ }^{2} \mathrm{p}$ values for the Chi-square tests

between G0 that had significantly higher scores in all domains in comparison to the other groups (Fig. 2). G1 and G2 had smaller numerical mean differences than with G0, but G1 had smaller scores in all categories. Moreover, significant differences were found in the following domains: foot pain (for $\mathrm{G} 0 \times \mathrm{G} 1$ and $\mathrm{G0} \times \mathrm{G} 2$ ), foot function (for $\mathrm{G} 0 \times \mathrm{G} 1$ and $\mathrm{G} 0 \times \mathrm{G} 2$ ), general foot health (for $\mathrm{G} 0 \times \mathrm{G} 1$ ), and footwear (for $\mathrm{G} 0 \times \mathrm{G} 1$ and G0 $\times$ G2) (Fig. 2).

The patients in G1 and G2 had smaller hallux strength values than the patients in G0; these variables indicated ulcer formation risks. However, there were no statistical differences between the ulcer risk groups in terms of toe strength ( $p$ was 0.05 ). The hallux strengths of $\mathrm{G} 2$ patients were significantly lower than the hallux strengths of G0 patients (Fig. 3). Moreover, the hallux strengths decreased as ulcer risks increased.

In summary, moderate differences were found between G0 and G1 and G2 for foot pain, foot function, general foot health, and footwear. There was a small but significant difference between G0 and G2 in regards to hallux strength. No significant differences were found between G1 and G2 for any of the variables (Table 2).

\section{Discussion}

Determining effective prevention strategies for people with DM and DPN involves a comprehensive description of the population in question regarding deficits in sensitivity, musculoskeletal properties, and function. This study investigated the foot health, toe and hallux strength levels, and foot function of people with DM based on the IWGDF's ulcer risk classifications [5]. Significant differences were observed in the function levels of the different classifications, and G2 had significantly lower hallux strengths than G0.

An interesting finding is that G1, which comprised patients with DPN, had the highest functional deficits, and G2, which comprised patients with DPN and foot deformities and/or vascular disease, presented the lowest strength of the hallux flexor and toes flexor muscles. The DPN severity may be a determining factor for which G1 presents the greatest impairments in function. Patients with foot deformities (G2) presented lower levels of muscle strength, but it is still not clear in the literature whether a decrease in muscle strength may be responsible for the development of foot deformities or the result [42]. The fact that G2 is composed of younger patients with foot deformities and lower strength levels, and G0 is composed of older patients without DPN, may be somewhat specific to the population of this study. Although the mean age of the 

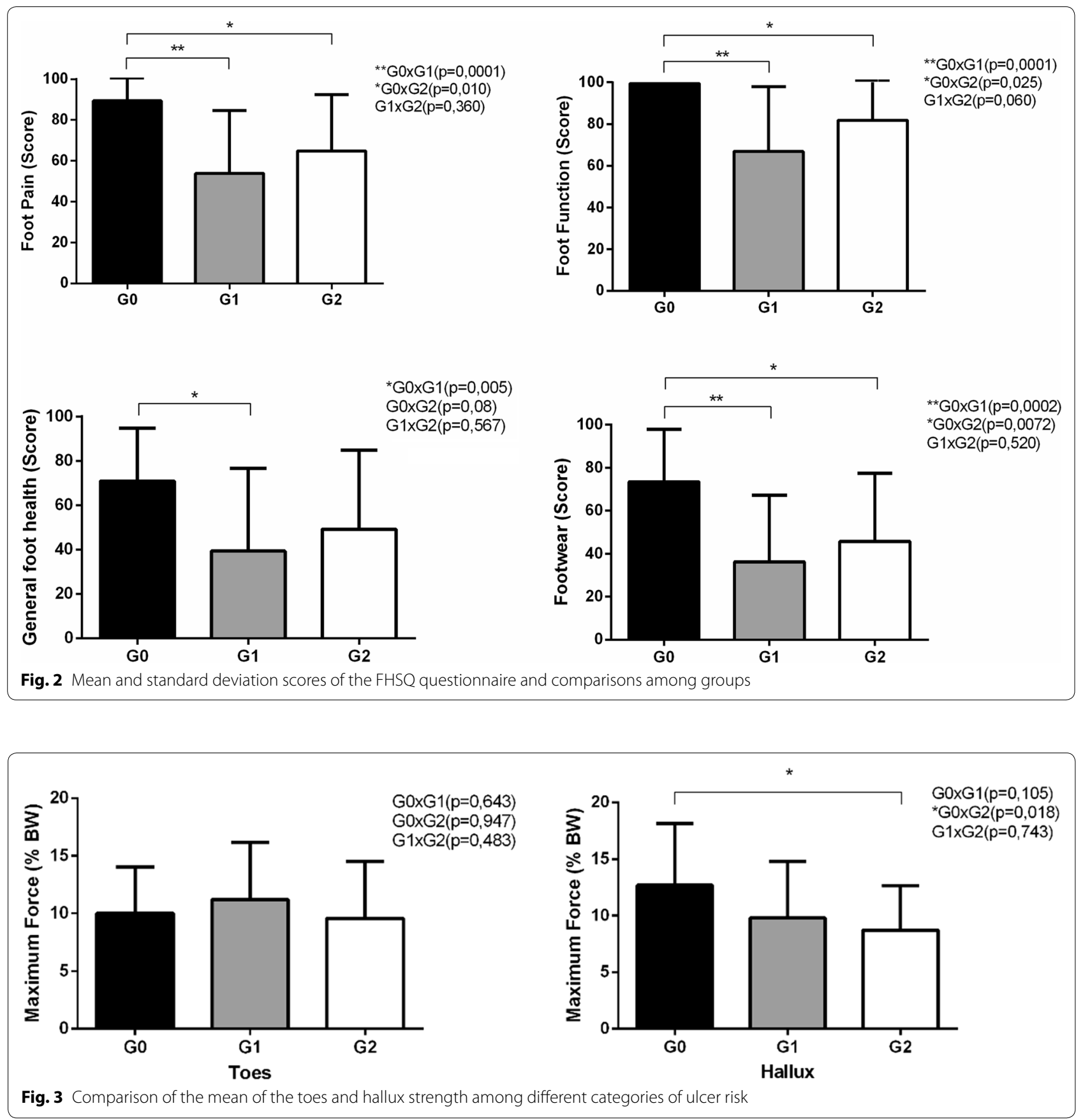

Table 2 Mean difference between groups and 95\% confidence interval ( $\mathrm{Cl}$ ) for all variables

\begin{tabular}{llll}
\hline & $\mathbf{G 0} \times \mathbf{~ G 1}$ mean diff $(\mathbf{9 5} \% \mathbf{~ C l})$ & $\mathbf{G 0} \times \mathbf{G} 2$ mean diff $(\mathbf{9 5} \% \mathbf{C l})$ & $\mathbf{G 1} \times \mathbf{G 2}$ mean diff $(\mathbf{9 5} \% \mathbf{C l})$ \\
\hline Foot pain (score) & $35.4(16.3$ to 54.6$)$ & $24.5(4.9$ to 44.0$)$ & $-10.9(-30.0$ to 8.1$)$ \\
Foot function (score) & $32.5(17.0$ to 48.1$)$ & $17.6(1.7$ to 33.4$)$ & $-14.9(-30.5$ to 0.5$)$ \\
General foot health (score) & $31.5(8.1$ to 54.8$)$ & $21.5(-2.2$ to 45.4$)$ & $-9.9(-33.2$ to 13.4$)$ \\
Footwear (score) & $37.2(16.4$ to 57.9$)$ & $27.7(96.5$ to 48.9$)$ & $-9.4(-30.2$ to 11.2$)$ \\
Toes (\% BW) & $-1.1(-4.3$ to 1.9$)$ & $0.4(-2.8$ to 3.6) & $1.6(-1.7$ to 4.9$)$ \\
Hallux (\% BW) & $2.8(-0.4$ to 6.2$)$ & $3.9(0.5$ to 7.3$)$ & $1.0(-2.4$ to 4.5$)$ \\
\hline
\end{tabular}

$B W$ body weight 
groups were not statistically different and could not be considered a bias in the results, the self-care strategies adopted by each patient, that unfortunately could not be controlled, may have influenced the function and strength level within groups.

The differences observed between groups in function (i.e., foot function, foot pain, general foot health, and footwear) showed that G0 had better outcomes than G1 and G2. Several studies support that patients with DPN (G1 and G2) have larger functional losses [43-45]. Some studies found that patients with DPN had higher levels of pain than patients without DPN [46-48]. It has also been shown that people with DM and DPN presented a decreased muscle strength and sensitivity that are associated to impairments in everyday locomotor activities [33]; such as reduced gait velocities, muscle activity alteration [49], lower limb kinematic pattern changes [50], and balance impairments [51-53]; however causality is difficult to specify. DPN patients also present loss of muscle function [54] by a manual muscle testing that may lead to functional losses. All these functional impairments made it difficult for these patients to perform daily living activities, can compromise the patient's physical and mental health, and negatively impact his or her quality of life [55].

G1 and G2 patients had lower hallux strengths than G0 patients. A possible reason for this is that the flexor hallucis longus and brevis have a smaller cross-sectional area than the flexor digitorum longus and brevis [56]. Thus, a minimal atrophy can lead to a significant reduction in force. Unlike the flexor muscle of the toes due the latter's greater cross-sectional area and, consequently, greater force generation. Although there has been no research similar to this study involving the foot strength of patients with different ulcer risk classifications, some studies have shown that the deterioration of the intrinsic foot muscles associated with low ranges of ankle motion might be a primary factor in the development of foot deformities [28]. Furthermore, toes deformity would put the intrinsic and extrinsic foot muscles at a poorer mechanical advantage, changing their fascicle length and lever arms, thus less force would be generated [57], just like we found in this study for strength levels in G2 (patients with foot deformities) compared to G0. Either way, as a cross sectional study, causality cannot be assessed to determine the causes of the lower strength levels in G2.

In people with DPN, Andersen et al. $[33,58]$ and Bus et al. [42] found atrophied foot muscles and increasing severity of DPN was associated with low ankle muscle strength that was proportional to muscular atrophy levels. A similar study found that decreased ankle strength was progressive and was more pronounced in older age groups than in younger age groups [59]. These findings corroborate this study's findings, including the observations that G2 had lower hallux strength than G0 and G1.

In a prior study, the atrophy of diabetic foot muscles was verified in a subclinical stage of DPN before the development of clinical DPN symptoms [60]. This showed that predicting/assessing foot strength and function of individuals with DM, and following such predictions with early interventions, could be a viable strategy to prevent the accelerated loss of muscle strength and to reduce the risk of developing ulcers in patients. This strategy could contribute to a better quality of life for these individuals.

This study had some limitations; first, the physical activity levels and the rehabilitation practices of the subjects could not be controlled. Second, there was no control group, and this prevented further conclusions regarding the consequences of foot function, foot strength, DM, and DPN progression. Finally, we emphasize that G3, defined by the IWGDF risk stratification system, was not included in this study due to the availability of these type of patient in the study setting, limiting our conclusions on muscle strength and foot function in more severe patients. Further studies should account for that when recruiting patients in different settings to include a broader range of risk categories.

Future studies should overcome these limitations; that is, they should reduce the heterogeneity of groups and include control groups. Further studies should also aim to improve the IWGDF's ulcer risk classifications by considering analyses of the foot strength levels and foot function of people with DM and DPN.

\section{Conclusion}

The foot function, hallux strengths, and foot health of people with DM and DPN differ by ulcer risk classification. These characteristics must be considered when evaluating and developing treatments and preventative strategies for such patients. In particular, patients with risk 2 classifications are more severely impaired in regards to foot strength and foot function than other patients. Early assessments of lower limb musculoskeletal deficits could potentially prevent changes to functions that occur with the progression of DM.

\section{Abbreviations}

IWGDF: International Working Group on the Diabetic Foot; CNPq: National Council for Scientific and Technological Development; CAPES: Agency Coordination of Improvement of Higher Education Personnel; DM: diabetes mellitus; DPN: diabetic polyneuropathy; G0: Group 0; G1: Group 1; G2: Group 2; FHSQ BR: Brazilian version of Foot Health Status Questionnaire; ANAD: National Association of Assistance to Diabetics; ICC: Intraclass Correlation Coefficient; BW: body weight. 


\section{Acknowledgements}

The authors are grateful to the State of São Paulo Research Foundation, Agency Coordination of Improvement of Higher Education Personnel (CAPES) and National Council for Scientific and Technological Development (CNPq) for the funding granted to this study.

\section{Authors' contributions}

JPP is responsible for the study design, intervention, interpretation of the data, writing the report and submission of the manuscript. JSSPF, EQS, RLM and $\mathrm{RHCJ}$ are responsible for the study design, data collection, management, analysis, and interpretation, writing the report and submission of the manuscript. ICNS is responsible for the study design, interpretation of the data, writing the report and submission of the manuscript. All authors read and approved the final manuscript.

\section{Funding}

Monteiro and Ferreira are funded by FAPESP [2019/02522-0; 2017/178483, respectively]; Sacco is a fellow by the National Council for Scientific and Technological Development (CNPq) Brazil (Process: 304124/2018-4); Queiroz and Cruvinel are funded by Agency Coordination of Improvement of Higher Education Personnel (CAPES, financial code 001). The funders do not have any role in the study and do not have any authority over any study activity or in the decision to submit the report for publication.

\section{Availability of data and materials}

All personal data from potential or enrolled patients will be maintained confidential before, during and after the study by encoding participant's name. All data access and storage are in keeping with National Health and Medical Research Council guidelines, as approved.

\section{Ethics approval and consent to participate}

This trial was approved by the Ethics Committee of the School of Medicine of the University of São Paulo (Protocol 075/16). All patients will be asked for written informed consent according to the standard forms and the researcher will obtain them.

\section{Consent for publication}

Written informed consent for publication of all images was obtained from the models.

\section{Competing interests}

The authors affirm that this study has not received any funding/assistance from a commercial organization which may lead to a conflict of interests.

\section{Author details}

${ }^{1}$ Department of Physical Therapy, Speech, and Occupational Therapy, School of Medicine, University of São Paulo, Rua Cipotânea, 51-Cidade Universitária, São Paulo 05360-160, Brazil. ${ }^{2}$ Departamento de Ciências da Saúde, Universidade Federal do Amapá, Macapá, Amapá, Brazil.

Received: 5 July 2019 Accepted: 22 October 2019 Published online: 30 October 2019

\section{References}

1. Hicks CW, Selvarajah S, Mathioudakis N, Perler BA, Freischlag JA, Black JH, et al. Trends and determinants of costs associated with the inpatient care of diabetic foot ulcers. J Vasc Surg. 2014;60(5):1247-1254.e2. https://doi. org/10.1016/j.jvs.2014.05.009.

2. Boulton AJM. Diabetic neuropathy and foot complications [Internet]. In: Handbook of clinical neurology, 1st ed. vol. 126. Elsevier B.V.; 2014. p. 97-107. http://dx.doi.org/10.1016/B978-0-444-53480-4.00008-4.

3. Waaijman R, De Haart M, Arts MLJ, Wever D, Verlouw AJWE, Nollet F, et al. Risk factors for plantar foot ulcer recurrence in neuropathic diabetic patients. Diab Care. 2014;37(6):1697-705

4. Boulton AJM, Valensi P, Tesfaye S. International Neuropathy Workshop of 2009: introduction to the final reports. Diab Metab Res Rev. 2011;27(2011):617-9.
5. Bus SA, van Netten JJ, Lavery LA, Monteiro-Soares M, Rasmussen A, Jubiz Y, et al. IWGDF guidance on the prevention of foot ulcers in at-risk patients with diabetes. Diabetes Metab Res Rev. 2016;32(1):16-24.

6. Veves A, Pham H, Armstrong D, Harvey C, Harkless L, Giurini J. Screening techniques to identify people at high risk for diabetic foot ulceration: a prospective multicenter trial. Diab Care. 2000;23(5):606 (Screening techniques to identify people at high risk for diabetic foot ulceration: a prospective multicenter trial).

7. Gordois A, Scuffham P, Shearer A, Oglesby A, Tobian JA. The health care costs of diabetic. Diab Care. 2003;26(6):1790-5.

8. Boulton AJM. The diabetic foot: From art to science. The 18th Camillo Golgi lecture. Diabetologia. 2004;47(8):1343-53.

9. Bruun C, Siersma V, Guassora AD, Holstein P, de Fine Olivarius N. Amputations and foot ulcers in patients newly diagnosed with type 2 diabetes mellitus and observed for 19 years. The role of age, gender and comorbidity. Diabet Med. 2013;30(8):964-72.

10. Shun CT, Chang YC, Wu HP, Hsieh SC, Lin WM, Lin YH, et al. Skin denervation in type 2 diabetes: correlations with diabetic duration and functional impairments. Brain. 2004;127(7):1593-605.

11. Sacco ICN, Picon AP, Macedo DO, Butugan MK, Watari R, Sartor CD. Alterations in the lower limb joint moments precede the peripheral neuropathy diagnosis in diabetes patients. 2015;17(6):1-8.

12. Watari R, Sartor CD, Picon AP, Butugan MK, Amorim CF, Ortega NRS, et al. Effect of diabetic neuropathy severity classified by a fuzzy model in muscle dynamics during gait. J Neuroeng Rehabil. 2014;11(1):1-9.

13. Gomes AA, Onodera AN, Otuzi MEI, Pripas D, Mezzarane RA, Sacco ICN. Electromyography and kinematic changes of gait cycle at different cadences in diabetic neuropathic individuals. Muscle Nerve. 2011;44(2):258-68.

14. Sacco ICN, Hamamoto AN, Gomes AA, Onodera AN, Hirata RP, Hennig EM. Role of ankle mobility in foot rollover during gait in individuals with diabetic neuropathy. Clin Biomech. 2009;24(8):687-92. https://doi. org/10.1016/j.clinbiomech.2009.05.003.

15. Giacomozzi C, Caselli A, Macellari V, Giurato L, Lardieri L, Uccioli L. Walking strategy in diabetic patients with peripheral neuropathy. Diab Care. 2002;25(8):1451-7.

16. Gomes AA, Ackermann M, Ferreira JP, Orselli MIV, Sacco ICN. Muscle force distribution of the lower limbs during walking in diabetic individuals with and without polyneuropathy. J Neuroeng Rehabil. 2017;14(1):1-13.

17. Guney A, Vatansever F, Karaman I, Kafadar IH, Oner M, Turk CY. Biomechanical properties of achilles tendon in diabetic vs non-diabetic patients. Exp Clin Endocrinol Diabetes. 2015;123(7):428-32. https://doi. org/10.1055/s-0035.

18. Błażkiewicz M, Sundar L, Healy A, Ramachandran A, Chockalingam N, Naemi R. Assessment of lower leg muscle force distribution during isometric ankle dorsi and plantar flexion in patients with diabetes: a preliminary study. J Diab Complications. 2015;2015(29):282-7.

19. Gautieri A, Passini FS, Silván U, Guizar-Sicairos M, Carimati G, Volpi P, et al. Advanced glycation end-products: Mechanics of aged collagen from molecule to tissue. Matrix Biol. 2017;59:95-108. https://doi.org/10.1016/j. matbio.2016.09.001.

20. Haus JM, Carrithers JA, Trappe SW, Trappe TA. Collagen, cross-linking, and advanced glycation end products in aging human skeletal muscle. J Appl Physiol. 2007;103(6):2068-76 http://www.ncbi.nlm.nih.gov/pubme d/17901242.

21. Atayde SA, Yoshinari NH, Nascimento DP, Catanozi S, Andrade PC, Velosa APP, et al. Experimental diabetes modulates collagen remodelling of joints in rats. Histol Histopathol. 2012;27(11):1471-9.

22. Andreassen TT, Seyer-Hansen K, Bailey AJ. Thermal stability, mechanical properties and reducible cross-links of rat tail tendon in experimental diabetes. Biochim Biophys Acta. 1981;677:313-7.

23. Vlassara H, Palace MR. Diabetes and advanced glycation end products. Diabetes Aging-related Complicat. 2017;201-12.

24. Paul RG, Bailey AJ. The effect of advanced glycation end-product formation upon cell-matrix interactions. Int J Biochem Cell Biol. 1999;31(6):653-60.

25. Lorimer AV, Hume PA. Stiffness as a risk factor for achilles tendon injury in running athletes. Sport Med. 2016;46(12):1921-38.

26. Lorentzen J, Kirk H, Fernandez-Lago H, Frisk R, Scharff Nielsen N, Jorsal M, et al. Treadmill training with an incline reduces ankle joint stiffness and 
improves active range of movement during gait in adults with cerebral palsy. Disabil Rehabil. 2017;39(10):987-93.

27. Semba RD, Bandinelli S. Relationship of an advanced glycation end product, plasma carboxymethyl-lysine, with slow walking speed in older adults: the InCHIANTI study. Eur J Appl Physiol. 2010;108(1):191-5. https:// doi.org/10.1007/s00421-009-1192-5.

28. Cheuy VA, Hastings MK, Commean PK, Mueller MJ. Muscle and joint factors associated with forefoot deformity in the diabetic neuropathic foot. Foot Ankle Int. 2016;37(5):514-21.

29. Crawford F, Inkster M, Kleijnen J, Fahey T. Predicting foot ulcers in patients with diabetes: a systematic review and meta-analysis. QJM. 2007;100(2):65-86.

30. Halawa MR, Eid YM, El-Hilaly RA, Abdelsalam MM, Amer AH. Relationship of planter pressure and glycemic control in type 2 diabetic patients with and without neuropathy. Diab Metab Syndr Clin Res Rev. 2018;12(2):99104. https://doi.org/10.1016/j.dsx.2017.09.010.

31. Bus SA, van Netten JJ. A shift in priority in diabetic foot care and research: $75 \%$ of foot ulcers are preventable. Diabetes Metab Res Rev. 2016;32:195-200.

32. Bus $S A$, Waaijman $R$. The value of reporting pressure-time integral data in addition to peak pressure data in studies on the diabetic foot: A systematic review. Clin Biomech. 2013;28(2):117-21. https://doi.org/10.1016/j. clinbiomech.2012.12.002.

33. Andersen Henning. Motor dysfunction in diabetes. Diab Metab Res Rev. 2012;26(6):89-92.

34. Plotnikoff RC, Lippke S, Karunamuni N, Eves N, Courneya KS, Sigal R, et al. Co-morbidity, functionality and time since diagnosis as predictors of physical activity in individuals with type 1 or type 2 diabetes. Diabetes Res Clin Pract. 2007:78(1):115-22.

35. MacGilchrist C, Paul L, Ellis BM, Howe TE, Kennon B, Godwin J. Lowerlimb risk factors for falls in people with diabetes mellitus. Diabet Med. 2010;27(2):162-8.

36. Partanen J, Niskanen L, Lehtinen J, Mervaala E, Siitonen O, Uusitupa M. Natural history of peripheral neuropathy in patients with non-insulindependent diabetes mellitus. N Engl J Med. 1995;333(2):89-94.

37. Ortega N, Picon A, Sacco I, Sartor C, Watari R. Classification of the severity of diabetic neuropathy: a new approach taking uncertainties into account using fuzzy logic. Clinics. 2012;67(2):151-6.

38. Boulton AJM, Armstrong DG, Albert SF, Frykberg RG, Hellman R, Kirkman MS, et al. Comprehensive foot examination and risk assessment. Diab Care. 2008;31(8):1679-85.

39. Ferreira AFB, Laurindo IMM, Rodrigues PT, Ferraz MB, Kowalski SC, Tanaka C. Brazilian version of the foot health status questionnaire (FHSQ-BR): cross-cultural adaptation and evaluation of measurement properties. Clinics. 2008;63(5):595-600.

40. Mickle KJ, Munro BJ, Lord SR, Menz HB, Steele JR. ISB Clinical Biomechanics Award 2009. Toe weakness and deformity increase the risk of falls in older people. Clin Biomech. 2009;24(10):787-91. https://doi. org/10.1016/j.clinbiomech.2009.08.011.

41. Erdfelder E, FAul F, Buchner A, Lang AG. Statistical power analyses using G*Power 3.1: tests for correlation and regression analyses. Behav Res Methods. 2009;41(4):1149-60.

42. Bus SA, Yang QX, Wang JH, Smith MB, Wunderlich R, Cavanagh PR. Intrinsic muscle atrophy and toe deformity in the diabetic neuropathic foot: a magnetic resonance imaging study. Diabetes Care. 2002;25(8):1444-50.

43. Parasoglou P, Rao S, Slade JM. Declining skeletal muscle function in diabetic peripheral neuropathy. Clin Ther. 2017;39(6):1085-103.

44. Allen MD, Doherty TJ, Rice CL, Kimpinski K. Physiology in Medicine: neuromuscular consequences of diabetic neuropathy. J Appl Physiol. 2016:121(1):1-6.
45. da Dibonaventura MC, Cappelleri JC, Joshi AV. Association between pain severity and health care resource use, health status, productivity and related costs in painful diabetic peripheral neuropathy patients. Pain Med. 2011;12(5):799-807.

46. Zhang Q, Zhang P, Yan R, Xu X, Mao C, Liu X, et al. A single-blinded trial using resting-state functional magnetic resonance imaging of brain activity in patients with type 2 diabetes and painful neuropathy. Diab Ther. 2019;10(1):135-47. https://doi.org/10.1007/s13300-018-0534-x.

47. Galer BS, Gianas A, Jensen MP. Painful diabetic polyneuropathy: epidemiology, pain description, and quality of life. Diabetes Res Clin Pract. 2000;47(2):123-8.

48. Gore M, Brandenburg NA, Dukes E, Hoffman DL, Tai KS, Stacey B. Pain severity in diabetic peripheral neuropathy is associated with patient functioning, symptom levels of anxiety and depression, and sleep. J Pain Symptom Manage. 2005;30(4):374-85.

49. Ilgin D, Savelberg HHCM, Meijer K, Willems PJB, Angin S, Schaper NC. Prolonged activity of knee extensors and dorsal flexors is associated with adaptations in gait in diabetes and diabetic polyneuropathy. Clin Biomech. 2010;25(5):468-75. https://doi.org/10.1016/j.clinbiomec h.2010.02.005.

50. Hazari A, Maiya AG, Shivashankara KN, Agouris I, Monteiro A, Jadhav R, et al. Kinetics and kinematics of diabetic foot in type 2 diabetes mellitus with and without peripheral neuropathy: a systematic review and metaanalysis. Springerplus. 2016;5(1):1819.

51. Allet L, Armand S, De Bie RA, Golay A, Monnin D, Aminian K, et al. The gait and balance of patients with diabetes can be improved: a randomised controlled trial. Diabetologia. 2010;53(3):458-66.

52. Turcot K, Allet L, Golay A, Hoffmeyer P, Armand S. Investigation of standing balance in diabetic patients with and without peripheral neuropathy using accelerometers. Clin Biomech. 2009;24(9):716-21.

53. Nardone A, Grasso M, Schieppati M. Balance control in peripheral neuropathy: are patients equally unstable under static and dynamic conditions? Gait Posture. 2006;23(3):364-73.

54. Sartor CD, Hasue RH, Cacciari LP, Butugan MK, Watari R, Pássaro AC, et al. Effects of strengthening, stretching and functional training on foot function in patients with diabetic neuropathy : results of a randomized controlled trial. BMC Musculoskelet Disord. 2014;15:1-13.

55. Pedras S, Carvalho R, Pereira MG. Predictors of quality of life in patients with diabetic foot ulcer: the role of anxiety, depression, and functionality. $J$ Health Psychol. 2018;23(11):1488-98.

56. Mickle KJ, Angin S, Crofts G, Nester CJ. Effects of age on strength and morphology of toe flexor muscles. J Orthop Sports Phys Ther. 2016;46(12):1065-70.

57. Bus SA, Maas M, Michels RPJ, Levi M. Role of intrinsic muscle atrophy in the etiology of claw toe deformity in diabetic neuropathy may not be as straightforward as widely believed. Diabetes Care. 2009;32(6):1063-7.

58. Andersen H, Nielsen S, Mogensen CE, Jakobsen J. Muscle strength in type 2 diabetes. Diabetes. 2004;53(6):1543-8. https://doi.org/10.2337/diabe tes.53.6.1543.

59. Andreassen CS, Jakobsen J, Andersen H. A progressive late complication in diabetic distal symmetric polyneuropathy. Diabetes. 2006;55:807-11.

60. Greenman RL, Khaodhiar L, Lima C, Dinh T, Giurini JM, Veves A. Foot small muscle atrophy is present before the detection of clinical neuropathy. Diabetes Care. 2005;28(6):1425-30.

\section{Publisher's Note}

Springer Nature remains neutral with regard to jurisdictional claims in published maps and institutional affiliations. 